\title{
Estructura estacional del ensamblaje de escarabajos coprófagos (Coleoptera: Scarabaeinae) en una parcela permanente de bosque seco tropical
}

\author{
Jorge Ari Noriega ${ }^{1}$ *, Willinton Barranco² ${ }^{2}$ José Hernández ${ }^{3}$, Elkin Hernández \\ Stephanie Castillo ${ }^{5}$, Daniel Monroy ${ }^{1}$, Héctor García ${ }^{5}$ \\ ${ }^{1}$ Laboratorio de Zoología y Ecología Acuática - LAZOEA, Universidad de Los Andes, Bogotá, Colombia \\ ${ }^{2}$ Vicerrectoría de Investigación, Universidad del Magdalena, Santa Marta, Colombia \\ ${ }^{3}$ Departamento Administrativo de Medio Ambiente - DADMA, Santa Marta, Colombia \\ ${ }^{4}$ Parques Nacionales Naturales, Territorial Caribe - Parque Tayrona, Santa Marta, Colombia \\ ${ }^{5}$ Herbario UTMC, Universidad del Magdalena, Santa Marta, Colombia
}

\begin{abstract}
Resumen
Los bosques secos tropicales en América del Sur son uno de los ecosistemas menos conocidos y presenta una de las mayores pérdidas de cobertura anual. Con el objetivo de entender la dinámica estacional de estos remanentes de bosque y generar herramientas de conservación, se ha propuesto la utilización de grupos indicadores como el de los escarabajos coprófagos. Durante las épocas de sequía y de lluvias en el 2012 se hizo un muestreo con el fin de conocer la estructura del ensamblaje de escarabajos coprófagos (Coleoptera: Scarabaeinae) en una parcela permanente de bosque seco tropical en la Universidad del Magdalena, distrito de Santa Marta (Magdalena, Colombia). Se instalaron tres transectos en la parcela con trampas de caída cebadas con excremento humano. Se registraron 5.001 individuos pertenecientes a 13 especies y siete géneros. Cabe resaltar la abundancia de la especie Canthon variabilis en las dos épocas. No se encontraron diferencias significativas en la abundancia entre las dos épocas, pero sí en la riqueza. De las 13 especies encontradas, 11 se registraron en la época de sequía y ocho en la de lluvias, en tanto que siete se encontraron exclusivamente en una u otra de las dos épocas. No se registró la presencia de Malagoniella astyanax ni de Digitonthophagus gazella, lo cual indica que el fragmento tiene un grado intermedio de conservación. El número de especies y la estructura del ensamblaje concordaron con lo registrado para la región Caribe, y se evidenció una marcada dinámica estacional. Es necesario continuar con este tipo de estudios y analizar el efecto funcional de la fragmentación y el tamaño de los fragmentos en los procesos y servicios ecosistémicos.
\end{abstract}

Palabras clave: conservación, dinámica estacional, época de sequía, parcela permanente, Scarabaeidae.

Seasonal structure of the assemblage of dung beetles (Coleoptera: Scarabaeinae) in a permanent plot of tropical dry forest

\begin{abstract}
Tropical dry forests in South America are one of the less known ecosystems and they exhibit one of the biggest annual losses in forest cover. From a conservationist perspective, it has been proposed to use indicator groups such as dung beetles to try to understand the seasonal dynamics of the species community structure of these forest remnants. Sampling was performed during the dry and rainy seasons in 2012 to characterize the species composition of the dung beetle assemblage (Coleoptera: Scarabaeinae) in a permanent plot of dry forest located in the University of Magdalena, Santa Marta district (Magdalena - Colombia). Three sampling transects were set up in the parcel using pitfall traps baited with human dung. A total of 5,001 individuals, corresponding to 13 species and seven genera were registered. The abundance of the species Canthon variabilis during the two seasons was noteworthy. Although no significant differences in abundance between seasons were found, they were registered for species richness. Of the 13 species that we found, eight appeared exclusively in one season. The absence of Malagoniella astyanax and Digitonthophagus gazella indicates that the parcel has an intermediate degree of conservation. The number of species and the structure of the assemblage matched with values of other studies carried out in the Caribbean region showing a strong seasonal dynamic. Our results highlight that it is necessary to continue with this type of studies, analyzing the functional effects of fragmentation and fragment size in ecosystem services and processes.
\end{abstract}

Key words: Conservation, dry season, permanent plot, Scarabaeidae, seasonal dynamics. 


\section{Introducción}

El bosque seco tropical (Bs-T) se define como aquella formación vegetal que presenta una cobertura boscosa continua y que se distribuye entre los 0 y los $1.000 \mathrm{~m}$ de altitud, en temperaturas superiores a los $24{ }^{\circ} \mathrm{C}$, con precipitaciones entre los $500 \mathrm{y}$ los $1.500 \mathrm{~mm}$ anuales y con uno o dos periodos de sequía al año (Pizano \& García, 2014). Debido a sus características biogeográficas y climáticas se le considera como una importante reserva de diversidad biológica (Espinal, 1985; Murphy \& Lugo, 1986; IAvH, 1998). Sin embargo, este tipo de bosque es uno de los ecosistemas más degradados, fragmentados y afectados por procesos antrópicos en América del Sur (Etter, 1993; IAvH, 1997; 1998; Fajardo, et al., 2005; Miles, et al., 2006). En Colombia solo persiste el $3 \%$ de su cobertura original debido a la expansión de la frontera agrícola y al pastoreo excesivo, lo que ha disminuido drásticamente su extensión original (IAvH, 1998). La región con mayor cobertura de los bosques secos tropicales en Colombia corresponde a la llanura del Caribe (6.046.376 ha), sin embargo, solo el 0,67 \% (41.000 ha) se encuentra dentro del sistema de protección de parques nacionales naturales (IAvH, 1998). Aunque mundialmente los bosques secos tropicales son uno de los ecosistemas que requieren mayor estudio, existen pocos trabajos detallados sobre su flora, fauna y ecología (Janzen, 1983; Murphy \& Lugo, 1986; Janzen, 1987; Prado \& Gibbs, 1993; Gentry, 1995; Prance, 2006; Pizano \& García, 2014).

Uno de los grupos de insectos más frecuentemente usados como bioindicadores en estudios de impacto y vigilancia ambiental son los escarabajos coprófagos (Coleoptera: Scarabaeinae), ya que responden de manera cualitativa y cuantitativa a la pérdida del hábitat y a los efectos de la fragmentación en diferentes ecosistemas (Davis, et al., 2001; Estrada \& Coates-Estrada, 2002; Halffter \& Arellano, 2002; McGeoch, et al., 2002; Boonrotpong, et al., 2004; Horgan, 2005; Shahabuddin, et al., 2005; Nichols, et al., 2007; Otavo, et al., 2013). Esta subfamilia contiene aproximadamente 6.000 especies a nivel mundial y en el territorio colombiano se han registrado cerca de 300 especies en 39 géneros (Medina, et al., 2001; Noriega, et al., 2007; Pulido, et al., 2007; Noriega, et al., 2008; Medina \& Pulido, 2009; Noriega, et al., 2011).

En el caso de los bosques secos tropicales, en varios trabajos se han utilizado los escarabajos coprófagos como bioindicadores de diferentes tipos de perturbaciones antrópicas (Escobar, 1997; 1998; Bustos-Gómez \& Lopera, 2003; Andresen, 2005; 2007; Martínez, et al., 2009; Barraza, et al., 2010). Sin embargo, en la región del cinturón árido pericaribeño (Hernández-Camacho, et al.,

\footnotetext{
*Correspondencia:

Jorge Ari Noriega, jnorieg@hotmail.com

Recibido: 2 de julio de 2015

Aceptado: 13 de enero de 2016
}

1992) se han hecho muy pocos estudios que hayan utilizado este grupo como indicador del estado de conservación, y la mayoría corresponden a reportes locales que no analizan la dinámica estacional (Noriega, et al., 2007; JiménezFerbans, et al., 2008; Martínez, et al., 2010; Navarro, et al., 2011; Solís, et al., 2011; Delgado-Gómez, et al., 2012). Por todo lo anterior, es necesario abordar estudios que no solo utilicen a los escarabajos como indicadores del estado de los fragmentos de bosque seco tropical, sino que, además, incluyan la variación en la estructura del ensamblaje en relación con su estacionalidad. En este trabajo se presenta un análisis estacional de la estructura del ensamblaje de escarabajos coprófagos en una parcela permanente de bosque seco tropical ubicada en la Universidad del Magdalena, Santa Marta, Colombia.

\section{Materiales y métodos}

\section{Área de estudio}

El estudio se hizo en una parcela de bosque seco tropical ubicada en el campus de la Universidad del Magdalena, en la ciudad de Santa Marta, departamento del Magdalena,

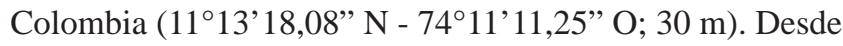
el año 1800 hasta principios de 1950 dicho campus hizo parte de un cultivo de caña de azúcar, el cual era muy común en toda la región. En 1970 se construyó la Universidad del Magdalena y desde su creación los relictos de bosque dentro del campus han sido retirados a machete, con máquinas y utilizando quemas controladas para establecer algunos monocultivos de algodón, maíz y hortalizas. A finales de los años 90 se suspendió el uso agrícola de estas áreas, y en 2007 se cedieron 3,5 hectáreas (parcela con $350.000 \mathrm{~m}^{2}$ de forma rectangular) de bosque seco tropical (Figura 1) al Programa de Biología de la Facultad de Ciencias Básicas para su conservación, recuperación e investigación (Strewe, et al., 2009).

La fisonomía y composición florística de la parcela permiten reconocer el zonobioma tropical alternohígrico (HernándezCamacho, et al., 1992), identificable por los vestigios de la cobertura vegetal original, que continúa hasta las estribaciones de la Sierra Nevada de Santa Marta. Esta formación puede encontrarse hasta los $1.000 \mathrm{~m}$ de altitud y se conoce como bosque seco tropical (IAvH, 1998), es decir, los llamados bosques higrotropofíticos (Dugand, 1973), bosques secos tropicales de Holdridge (1979) y bosque tropical deciduo por la sequía de baja altitud según la clasificación de la UNESCO (1973). Torrijos \& Cuadrado (2008) hicieron el inventario florístico de la Universidad del Magdalena y encontraron 84 especies distribuidas en 25 familias, incluidas las lianas, las herbáceas y los árboles. Posteriormente, en un par de trabajos de pregrado, Hernández-R (2012) y Rojano \& España (2013) registraron un promedio de 250 individuos con un diámetro a la altura del pecho (DAP) de 2,5 cm o más, correspondientes a 22 especies y 11 familias de plantas. En la actualidad se evidencia un proceso de regeneración 


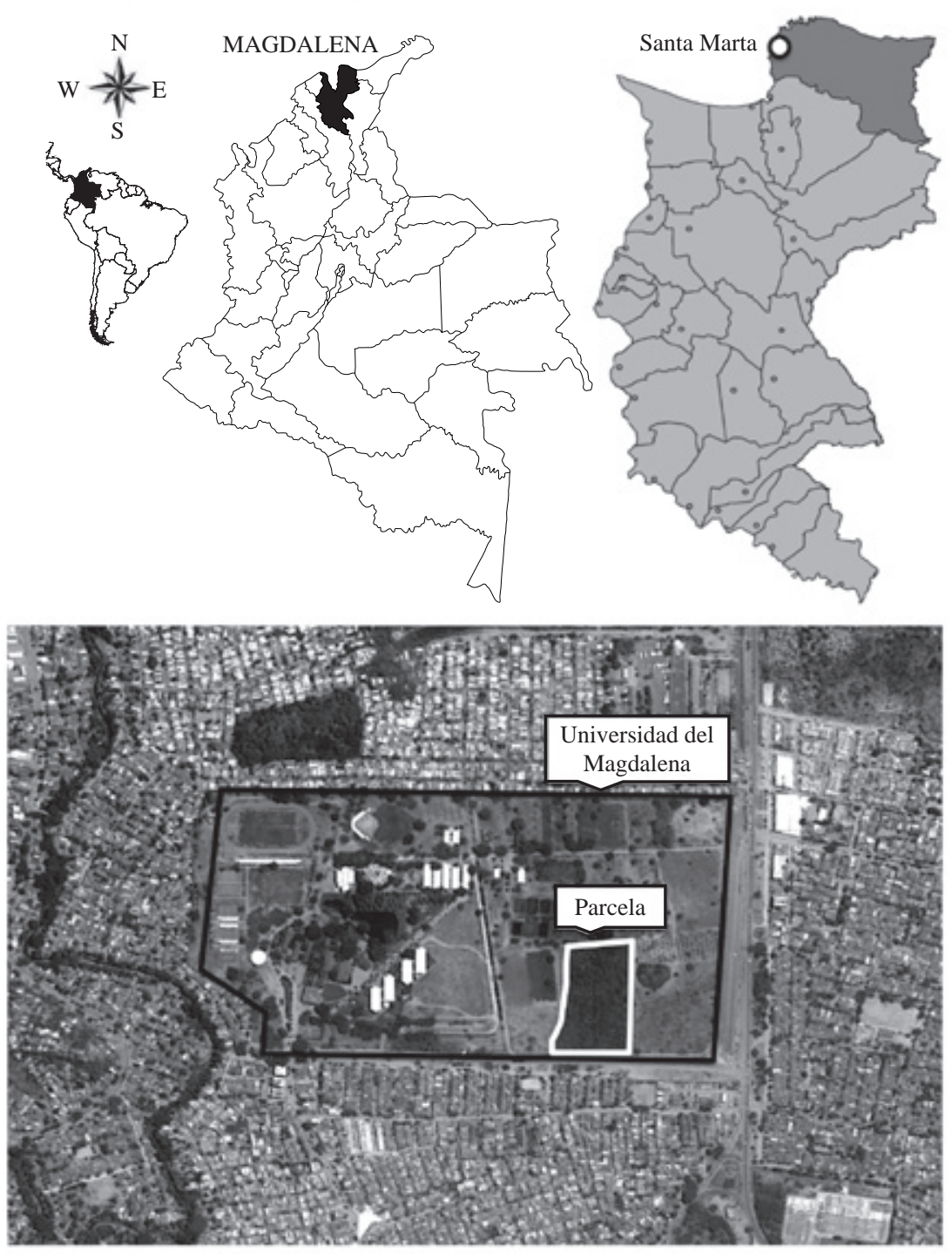

Figura 1. Ubicación de la parcela permanente de bosque seco tropical en la Universidad del Magdalena, Santa Marta, Colombia.

natural dominado por las familias Leguminosae, Malvaceae, Capparaceae, Euphorbiaceae, Poaceae y Apocynaceae, y se pueden encontrar elementos de la vegetación original, como los árboles olla de mono (Lecythis minor Jacq.), capote (Machaerium milleflorum Pittier), buche colorado (Pithecellobium roseum Spruce ex Benth.) y aruñagato (Pisonia aculeata L.), inmersos en rastrojos formados por lianas (Paullinia macrophylla Kunth, Serjania spp., Fridericia pubescens (L.) L.G.Lohmann, Luffa cylindrica (L.) M.Roem.), y arbustos leñosos (Capparis flexuosa (L.) L., Cordia alba (Jacq.) Roem. \& Schult., Calliandra magdalenae (DC.) Benth.) (E. Carbono, com. pers.)

La zona presenta un régimen de precipitación de tipo bimodal tetraestacional, con dos períodos de concentración de lluvias, uno de mayo a junio y otro de septiembre a noviembre, cuya mayor intensidad se registra en octubre, y dos períodos secos, de los cuales el más intenso va de diciembre a abril, y un veranillo de julio a agosto (Strewe, et al., 2009). La precipitación anual promedio es de $578 \mathrm{~mm}$, la temperatura promedio anual es de $27^{\circ} \mathrm{C}$, con una máxima de $32,6{ }^{\circ} \mathrm{C}$ y una mínima de $23,3{ }^{\circ} \mathrm{C}$, y una humedad relativa promedio de 74 \% (Rangel \& Garzón, 1995). Sobre la diversidad faunística en la parcela, los hallazgos de más de dos años de investigación incluyen 191 especies de aves (de las cuales 73 son migratorias), 15 especies de mamíferos, seis especies de anfibios y siete especies de reptiles (Strewe, et al., 2009, Montes-Correa, et al., 2015).

\section{Muestreo de los escarabajos coprófagos}

Se llevaron a cabo dos muestreos durante los meses de febrero y octubre de 2012, correspondientes a las dos épocas climáticas: sequía (baja pluviosidad) y lluvias (alta pluviosidad). El estudio consistió en establecer tres transectos separados $50 \mathrm{~m}$ entre sí; en cada transecto se colocaron durante 48 horas cinco trampas de caída con 
$30 \mathrm{~m}$ de distancia entre ellas y cebadas con excremento humano (Noriega \& Fagua, 2009). El material recolectado se identificó según la especie en las instalaciones del Laboratorio de Entomología de la Universidad del Magdalena con base en diferentes claves taxonómicas (Howden \& Young, 1981; Kohlmann \& Solis, 1997; Medina \& LoperaToro, 2000; Kohlmann \& Solis, 2001; Solis \& Kohlmann, 2002; 2004; Camero, 2010; Edmonds \& Zidek, 2010; Vaz-De-Mello, et al., 2011; Solis \& Kohlmann, 2013), así como en la comparación con material de la colección de referencia del primer autor (CJAN) y el apoyo de especialistas internacionales.

\section{Análisis de los datos}

Se hicieron curvas de acumulación de especies para cada una de las épocas climáticas utilizando el programa EstimateS v. 9.1 (Colwell, 2013), así como una prueba de normalidad con el test de Shapiro-Wilk y una prueba de Bartlett para evaluar la homogeneidad de las varianzas. El análisis de los datos arrojó una distribución no normal, por lo que se utilizó el test no paramétrico de Mann-Whitney para comparar la abundancia y la riqueza entre épocas. Todas las pruebas estadísticas se hicieron con el programa Statistix v. 8.1 empleando un nivel de confianza de $\alpha=0,05$.

\section{Resultados}

Las curvas de acumulación de especies demostraron que el muestreo fue eficiente para las dos épocas (de sequía y de lluvias), con un rango que osciló entre 80 y 93 \%, y que las curvas de singletons y doubletons se cruzaban y disminuían (Figura 2).

\section{Estructura del ensamblaje de escarabajos coprófagos}

Se recolectaron 5.001 individuos correspondientes a 13 especies, siete géneros y cinco tribus (Tabla 1). El género con mayor número de especies fue Canthon, con cinco especies, seguido de Onthophagus y Eurysternus, con dos especies cada uno; el resto de géneros presentó solamente una especie. La especie más abundante en el muestreo fue Canthon variabilis $(\mathrm{n}=3.696,73,8 \%)$, seguida por $C$. euchalceum $(\mathrm{n}=534,10,6 \%)$, C. juvencus $(\mathrm{n}=308,6,1 \%)$ y C. acutoides $(\mathrm{n}=254,5 \%)$. El ensamblaje presentó la estructura típica de los tres gremios de relocalización: de las 13 especies recolectadas seis tienen hábitos paracópridos (cavadores), cinco telecópridos (rodadores) y dos son endocópridas (residentes) (Tabla 1).

\section{Dinámica estacional del ensamblaje}

El mayor número de especies se obtuvo en la época de sequía, con 11 registros, mientras que en la época de lluvias solo se registraron ocho especies (Tabla 1). La especie que presentó la mayor abundancia en las dos épocas fue $C$. variabilis. La época con el mayor número de individuos fue el periodo de lluvias $(\mathrm{n}=2.551)$ y el menor se registró en la época de sequía ( $\mathrm{n}=2.450)$; aunque no se encontraron diferencias significativas en la abundancia entre las dos
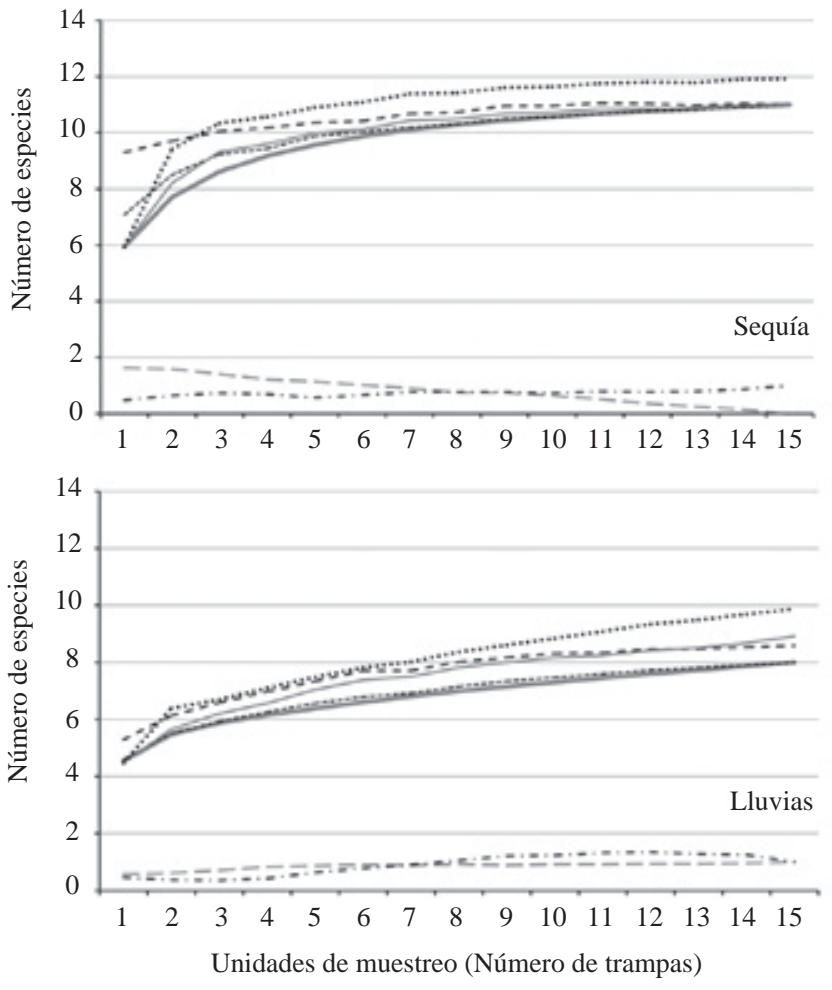

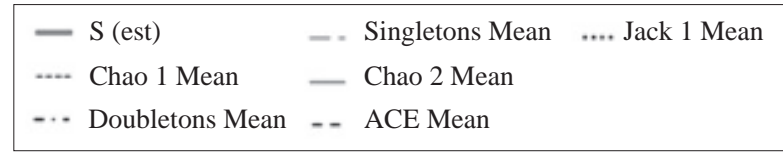

Figura 2. Curvas de acumulación de especies según las unidades de muestreo (número de trampas) para las dos épocas de muestreo (sequía y lluvias) en la parcela permanente de bosque seco tropical en la Universidad del Magdalena, Santa Marta, Colombia.

épocas (MW, $W=125,0, p=0,6186, n=15, \alpha=0,05$, Figura $3)$, sí se encontraron diferencias significativas en la riqueza (MW, $\mathrm{W}=53,5, \mathrm{p}=0,0127, \mathrm{n}=15, \alpha=0,05$, Figura 4).

En cuanto a la especificidad por época climática (sequía y lluvias), se encontró que seis de las 13 especies eran generalistas, pues se presentaron en las dos épocas, mientras que dos especies (Dichotomius belus y Coprophanaeus corythus) se registraron únicamente en la época de lluvias, y cinco especies (Eurysternus impressicollis, Eurysternus plebejus, Onthophagus hirculus, Onthophagus marginicollis y Uroxys macrocularis) en la época de sequía (Tabla 1). Los resultados evidenciaron que en esta localidad y fragmento de bosque seco tropical no se registró una relación entre los niveles de precipitación y la abundancia, pero sí se evidenció un efecto sobre la riqueza, así como un marcado recambio de especies entre épocas.

\section{Discusión}

\section{Estructura del ensamblaje de escarabajos coprófagos}

Teniendo en cuenta el tamaño reducido y la perturbación antrópica del fragmento, el número de especies registrado 
Tabla 1. Listado de las especies de la subfamilia Scarabaeinae (Coleoptera) y número de individuos en cada temporada de muestreo (sequía y lluvias) presentes en la parcela permanente de bosque seco tropical en la Universidad del Magdalena, Santa Marta, Colombia. Gremio de relocalización alimenticio (GRA, C: Cavador - paracóprido, R: Rodador - telecóprido y E: Residente - endocóprido; Tamaño: S > 10 mm y s $\leq 10 \mathrm{~mm}$ ).

\begin{tabular}{|c|c|c|c|c|c|c|}
\hline \multirow[t]{2}{*}{ Tribu } & \multirow[t]{2}{*}{ GRA } & \multirow[t]{2}{*}{ Especies } & & \multicolumn{2}{|c|}{ Número de individuos } & \multirow[t]{2}{*}{ Total } \\
\hline & & & & Sequía & Lluvias & \\
\hline Ateuchini & Cs & Uroxys macrocularis (Howden \& Young, 1981) & & 11 & 0 & 11 \\
\hline \multirow[t]{2}{*}{ Coprini } & Cs & Canthidium euchalceum Balthasar, 1939 & & 32 & 502 & 534 \\
\hline & CS & Dichotomius belus (Harold, 1880) & & 0 & 2 & 2 \\
\hline \multirow[t]{5}{*}{ Deltochilini } & Rs & Canthon acutoides Schmidt, 1922 & & 136 & 118 & 254 \\
\hline & Rs & Canthon juvencus (Harold, 1868) & & 209 & 99 & 308 \\
\hline & Rs & Canthon lituratus (Germar, 1813) & & 18 & 3 & 21 \\
\hline & Rs & Canthon mutabilis Lucas, 1857 & & 32 & 48 & 80 \\
\hline & Rs & Canthon variabilis (Martínez, 1948) & & 1.918 & 1.778 & 3.696 \\
\hline \multirow[t]{2}{*}{ Oniticellini } & Es & Eurysternus impresicollis Castelnau, 1840 & & 2 & 0 & 2 \\
\hline & Es & Eurysternus plebejus Harold, 1880 & & 4 & 0 & 4 \\
\hline \multirow[t]{2}{*}{ Onthophagini } & Cs & Onthophagus hirculus Mannerheim, 1829 & & 14 & 0 & 14 \\
\hline & Cs & Onthophagus marginicollis Harold, 1880 & & 74 & 0 & 74 \\
\hline \multirow[t]{2}{*}{ Phanaeini } & CS & Coprophanaeus corythus (Harold, 1863) & & 0 & 1 & 1 \\
\hline & & & Total & 2.450 & 2.551 & 5.001 \\
\hline
\end{tabular}

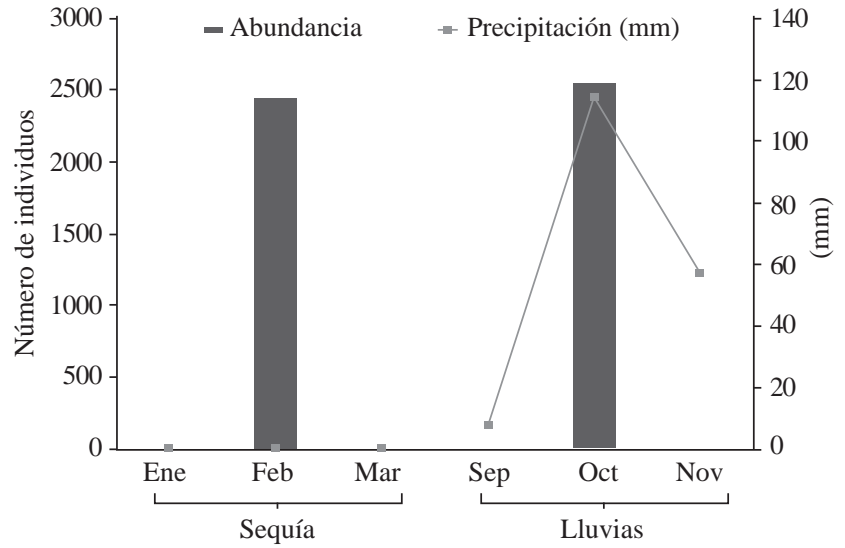

Figura 3. Variación de la abundancia de escarabajos coprófagos y de la precipitación durante las dos épocas de muestreo (sequía y lluvias) en la parcela permanente de bosque seco tropical en la Universidad del Magdalena, Santa Marta, Colombia.

para la zona fue representativo en comparación con otros estudios en el Caribe colombiano. Las especies capturadas correspondían a 22,8 \% de las registradas por Noriega, $\boldsymbol{e t}$ al. (2007) para la provincia de la Sierra Nevada de Santa Marta (SNSM), a $50 \%$ de las reportadas por JiménezFerbans, et al. (2008) en ambientes secos de la región de Santa Marta, a 44,8 \% de las reportadas por Martínez, et al. (2009) para la cuenca del río Gaira (SNSM) y a $59 \%$ de las especies reportadas por Barraza, et al. (2010) en Bahía Concha, lo cual indicaría que la zona mantiene una fauna típica de escarabajos coprófagos propia de los bosques secos tropicales de esta región del país (Noriega, et al. 2013).

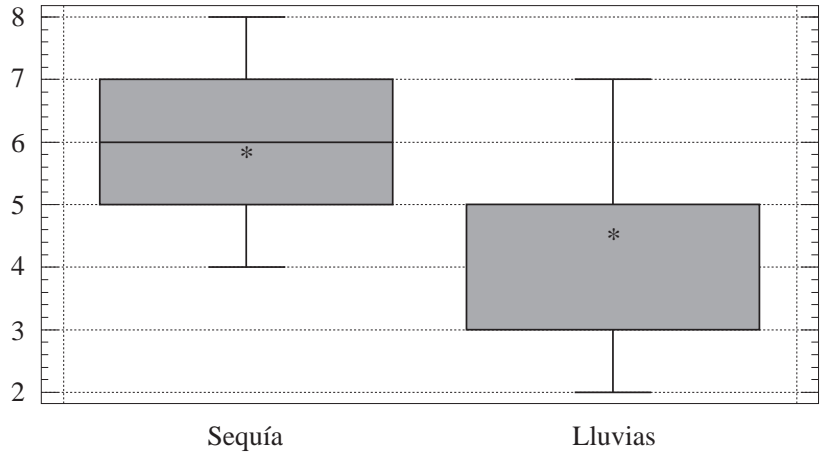

Figura 4. Diagrama de caja de la riqueza de especies de escarabajos coprófagos en las dos épocas de muestreo (sequía y lluvias) en la parcela permanente de bosque seco tropical en la Universidad del Magdalena, Santa Marta, Colombia.

El género Canthon se registró en las dos épocas, lo cual puede indicar su condición generalista y que potencialmente puede recurrir a estrategias que le permitan aprovechar los pocos recursos existentes (H. García, datos sin publicar). Estos datos coinciden con lo reportado por JiménezFerbans, et al. (2008), Martínez, et al. (2009, 2010) y Solís, et al. (2011), y sugieren que este género es uno de los más característicos de la región Caribe. Entre las especies menos abundantes se registró $C$. corythus, de la cual se recolectó un solo individuo en época de lluvias, lo que concuerda con la información registrada por otros autores en cuanto a esta especie, la cual se encuentra asociada a hábitats muy conservados, y se recolecta más frecuentemente en carroña (Gámez, et al., 2006; Edmonds \& Zidek, 2010). Además, 
el tamaño reducido del fragmento, su grado de perturbación y el hecho de que no se usó carroña como cebo en las trampas explicarían el bajo número de individuos de esta especie.

Es importante señalar la ausencia de una especie típica del bosque seco tropical como lo es Malagoniella astyanax, la que según los resultados de este estudio podría ser un excelente indicador de parches grandes de este tipo de bosque en la región. En contraste, la ausencia de la especie Digitonthophagus gazella es un indicador del buen nivel de recuperación de este fragmento de bosque, ya que aunque está reportada para la región (Rivera \& Wolff, 2007; Martínez, et al., 2009; Noriega, et al., 2011), se trata de una especie asociada a zonas abiertas o bosques demasiado perturbados.

\section{Dinámica estacional del ensamblaje}

A pesar de no haberse registrado diferencias significativas en la abundancia entre épocas, con base en otros muestreos en la zona se sugiere que la abundancia debe disminuir entre la época de lluvias y la época de sequía, lo que concuerda con lo reportado para otras localidades a nivel mundial (Janzen, 1983; Montes De Oca \& Halffter, 1995; Andresen, 2005; Quintero \& Halffter, 2009). Este patrón se debe a que el aumento de la humedad del suelo permite que los adultos emerjan cuando la bola-nido se ha ablandado (Martínez \& Montes de Oca, 1994). Igualmente, las precipitaciones ofrecen más recursos a nivel cualitativo y cuantitativo al proporcionar alimento a los vertebrados, con el consecuente aumento en la producción de excremento (Navarro, et al., 2011).

La aparición exclusiva de grandes paracópridos (CS, Tabla 1 ) como $D$. belus y $C$. corythus en época de lluvias, probablemente se relaciona con el hecho de que estas especies necesitarían una mayor cantidad de recursos y en esta época es posible que sea más fácil la construcción de túneles en el suelo debido a su menor compactación y a un mejor aprovechamiento del recurso que permanece húmedo por más tiempo (Halffter \& Favila, 1993). En este sentido, es probable que las especies asociadas a la época de sequía se hayan especializado en aprovechar pequeños recursos poco hidratados y en hacer nidos superficiales como lo hacen las especies rodadoras, pero comprobarlo requeriría estudios más profundos.

Escobar (2000) reconoce una estrecha relación entre los niveles de precipitación y la variación de las comunidades entre la época de sequía y la de lluvias en los bosques secos tropicales, en tanto que en los bosques de montaña los cambios en la abundancia de especies de escarabajos coprófagos no están relacionados con los niveles de precipitación. La segregación espacial entre las especies de escarabajos, documentada en otros estudios (Janzen, 1983; Bustos, et al., 2003; Andresen, 2005; Horgan, 2005; Quintero \& Halffter, 2009; Noriega, 2015), es un mecanismo que disminuye la competencia interespecífica y la presión por el recurso alimenticio, lo cual concuerda con lo encontrado en este estudio. De igual manera, las abundancias registradas fueron bajas y se mantuvieron en las dos épocas de muestreo debido al tamaño reducido de la parcela, a la escasez de recursos y a la baja conectividad espacial de la parcela con fragmentos cercanos que podrían permitir un flujo de individuos o de mamíferos que abastecieran de recurso a los escarabajos.

El presente trabajo representa un primer aporte al conocimiento de la fauna de Scarabaeinae de la región, y constituye un estudio de base para posteriores análisis de la dinámica temporal de pequeños fragmentos de bosque seco en el Caribe colombiano. A manera de sugerencia, sería fundamental continuar con esta línea de estudios en la región, incluida la evaluación de aspectos funcionales, lo que requeriría aumentar los esfuerzos de recolección para cubrir la totalidad del año, usar otros tipos de cebos como carroña y diplópodos, replicar el estudio en otros fragmentos cercanos con diferentes tamaños y priorizar las campañas de conservación y restauración de los pocos relictos de bosque que aún existen en la región.

\section{Agradecimientos}

Al Programa de Biología de la Facultad de Ciencias Básicas y a la Vicerrectoría de Investigación de la Universidad del Magdalena. Al Laboratorio de Zoología y Ecología Acuática - LAZOEA, de la Universidad de Los Andes. A Eduino Carbono por la información sobre la estructura de la vegetación de la parcela. A Darren Mann y a Fernando Vaz-De-Mello por su valiosa ayuda en la confirmación del material recolectado. A David Morris y Javier Santos por la revisión crítica del resumen en inglés.

\section{Conflicto de intereses}

Los autores declaran que no tienen conflicto de intereses.

\section{Bibliografía}

Andresen, E. (2003). Effect of forest fragmentation on dung beetle communities and functional consequences for plant regeneration. Ecography 26: 87-97.

Andresen, E. (2005). Effects of season and vegetation type on community organization of dung beetles in a tropical dry forest. Biotropica 37: 291-300.

Andresen, E. (2007). Dung beetle assemblages in primary forest and disturbed habitats in a tropical dry forest landscape in western Mexico. Journal of Insect Conservation 12 (6): 639-650.

Barraza, J., Montes, J., Martínez, N., Deloya, C. (2010). Ensamblaje de escarabajos coprófagos (Scarabaeidae: Scarabaeinae) del bosque tropical seco, Bahía Concha, Santa Marta (Colombia). Revista Colombiana de Entomología. 36 (2): 285-291.

Boonrotpong, S., Sotthibandhu, S., Pholpunthin, C. (2004). Species composition of dung beetles in the primary and secondary forests at Ton Nga Chang Wildlife Sanctuary. ScienceAsia. 30: 59-65. 
Bustos, F., Gómez, L., Lopera, A. (2003). Preferencia por el cebo de los escarabajos coprófagos (Coleóptera: Scarabaeidae: Scarabaeinae) de un remanente de bosque seco tropical al norte del Tolima (COLOMBIA). Monografías Tercer Milenio, SEA, Zaragoza 3: 59-65.

Camero, E. (2010). Los escarabajos del género Eurysternus Dalman, 1824 (Coleoptera: Scarabaeidae) de Colombia. Boletín de la Sociedad Entomológica Aragonesa (S.E.A.). 46: $147-179$.

Colwell, R. K. (2013). EstimateS: Statistical estimation of species richness and shared species from samples. Version 9.1. (Software and User's Guide). URL: purl.oclc.org/estimates.

Davis, A. J., Holloway, J. D., Huijbregts, H., Krikken, J., KirkSpringgs, A., H., Sutton, S. L. (2001). Dung beetles as indicators of change in the forests of northern Borneo. Journal of Applied Ecology. 38: 593-616.

Delgado-Gómez, P., Lopera, A., Rangel-Ch., J. O. (2012). Variación espacial del ensamblaje de escarabajos coprófagos (Scarabaeidae: Scarabaeinae) en remanentes de bosque seco en Chimichagua (Cesar, Colombia). En: J.O. Rangel-Ch. (Ed.), Colombia Diversidad Biótica XII: La región Caribe de Colombia. Instituto de Ciencias Naturales, Bogotá. p. 833-849.

Dugand, A. (1973). Elementos para un curso de geobotánica en Colombia. Cespedesia. 2 (6-8): 137-479.

Edmonds, W. \& Zidek, J. (2010). A taxonomic review of the Neotropical genus Coprophanaeus Olsoufieff, 1924 (Coleoptera: Scarabaeidae, Scarabaeinae). Insecta Mundi. 0129: 1-111.

Escobar, F. (1997). Estudio de la comunidad de coleópteros coprófagos (Scarabaeidae) en un remanente de bosque seco al norte del Tolima, Colombia. Caldasia 19: 419-430.

Escobar, F. (1998). Análisis regional de la comunidad de escarabajos coprófagos (Coleóptera: Scarabaeidae: Scarabaeine) de los bosques secos de la región Caribe de Colombia. En: Informe Nacional sobre el Estado de la Biodiversidad 1997- Colombia. María Elfi Chávez y Natalia Arango (Ed.). Instituto Humboldt, PNUMA, Ministerio del Medio Ambiente. Santafé de Bogotá: 1 vol. p. 72-75.

Escobar, F. (2000). Diversidad de coleópteros coprófagos (Scarabaeidae: Scarabaeinae) en un mosaico de hábitats en la Reserva Natural Nukak, Guaviare, Colombia. Acta Zoologica Mexicana. 79: 103-121.

Espinal, L. S. (1985). Geografía ecológica del departamento de Antioquia. Revista de la Facultad Nacional de Agronomía. 38 (1): 24-39.

Estrada, A. \& Coates-Estrada, R. (2002). Dung beetles in continuous forest, forest fragments and in an agricultural mosaic habitat island at Los Tuxtlas, Mexico. Biodiversity and Conservation. 11: 1903-1918.

Etter, A. (1993). Diversidad ecosistémica en Colombia hoy. En: Nuestra Diversidad Biológica, CEREC (Ed.), Fundación Alejandro Ángel Escobar, Bogotá. p. 47-66.

Fajardo, L., Gonzáles, V., Nassar, J., Lacabana, P., Portillo, C. A., Carrasquel, F., Rodríguez, J. P. (2005). Tropical dry forests of Venezuela: Characterization and current conservation status. Biotropica. 37: 531-546.

Gentry, A. H. (1995). Diversity and floristic composition of neotropical dry forest. En: Tropical deciduous forest ecosystem. S. Bullock, E. Medina \& H. A. Mooney (Eds). Cambridge Univ. Press, Cambridge. p. 116-194.

Halffter, G. \& Favila, M. (1993). The Scarabaeinae (insecta: Coleoptera), an animal group for analyzing, inventorying and monitoring biodiversity in tropical rainforest and modified landscapes. Biology international. 27: 15-21.

Halffter, G. \& Arellano, L. (2002). Response of dung beetle diversity to human-induced changes in a tropical landscape. Biotropica. 34 (1): 144-154.

Hernández-Camacho, J., Hurtado, A., Ortiz, R., Walschburger, T. (1992). Unidades biogeográficas de Colombia. En: Halffter, G. (Ed.) La diversidad biológica de Iberoamérica I. Acta Zoológica Mexicana. p. 55-151.

Hernández-R., J. (2012). Informe de las actividades de apoyo profesional para el levantamiento de la vegetación en la parcela permanente de bosque seco tropical del campus de la Universidad del Magdalena. Facultad de Ciencias Básicas, Universidad del Magdalena. 45 p.

Holdridge, L. (1979). Ecología basada en zonas de vida. Instituto Interamericano de Ciencias Agrícolas San José, Costa Rica. $159 \mathrm{p}$.

Horgan, F. G. (2005). Aggregated distribution of resources creates competition refuges for rainforest dung beetles. Ecography. 28: 603-618.

Howden, H. F. \& Young, O. P. (1981). Panamian Scarabaeinae: Taxonomy, distribution, and habits (Coleoptera, Scarabaeidae). Contributions of the American Entomological Institute. 18 (1): 1-204.

Instituto Alexander von Humboldt (IAVH). (1997). Caracterización ecológica de cuatro remanentes de bosque seco tropical de la región Caribe colombiana. Grupo de Exploraciones Ecológicas Rápidas, IAVH, Villa de Leyva. 76 p.

Instituto Alexander von Humboldt (IAvH). (1998). El bosque seco tropical (Bs-T) en Colombia. En: M. Chávez y N. Arango (Eds.) Informe nacional sobre el estado de la biodiversidad de Colombia, 1997. Programa de inventarios de la biodiversidad. Grupo de exploración y monitoreo, GEMA. Ministerio del Medio Ambiente-Naciones Unidas, Bogotá. 215 p.

Janzen, D. (1983). Seasonal changes in abundance of large nocturnal dung beetles (Scarabaeidae) in Costa Rica deciduous forest and adjacent horse pasture. Oikos. 33: 274-283.

Janzen, D. H. (1987). Insect diversity of a Costa Rican dry forest: Why keep it. Biol. Journal of the Linn. Soc. 30: 343-356.

Jiménez-Ferbans, L., Mendieta, W., García, H., Amat, G. (2008). Notas sobre los escarabajos coprófagos (Coleoptera: Scarabaeinae) en ambientes secos de la región de Santa Marta, Colombia. Acta Biológica Colombiana. 13 (2): 203-208. 
Kohlmann, B. \& Solís, A. (1997). El género Dichotomius (Coleoptera: Scarabaeidae) en Costa Rica. Giornale Italiano di Entomologia. 8: 343-382.

Kohlmann, B. \& Solís, A. (2001). El género Onthophagus (Coleoptera: Scarabaeidae) en Costa Rica. Giornale Italiano di Entomologia. 49 (9): 159-261.

Martínez, I. \& Montes de Oca, E. (1994). Observaciones sobre algunos factores microambientales y el ciclo biológico de dos especies de escarabajos coprófagos (Coleoptera: Scarabaeidae: Canthon). Folia Entomológica Mexicana. 91: 47-59.

Martínez, N. J., García, H., Pulido, L. A., Ospino, D. D., Narváez, J. C. (2009). Escarabajos coprófagos (Coleoptera: Scarabaeinae) de la vertiente noroccidental de la Sierra Nevada de Santa Marta, Colombia. Neotropical Entomology. 38 (6): 708-715.

Martínez, N. J., Cañas, L. M., Rangel, J. L., Barraza, J. M., Montes, J. M., Blanco, O. R. (2010a). Coleópteros coprófagos (Scarabaeidae: Scarabaeinae) en un fragmento de bosque seco tropical en el departamento del Atlántico, Colombia. Boletín del Museo de Entomología de la Universidad del Valle. 11 (1): 21-30.

Martínez, N. J., Cañas, L. M., Rangel, J. L., Blanco, O., Mendoza, J. D., Cohen, S. (2010b). Coleópteros coprófagos (Scarabaeidae: Scarabaeinae) en la Reserva Natural Las Delicias (RND), Sierra Nevada de Santa Marta (SNSM), Colombia. Boletín Científico Museo de Historia Natural. 14 (2): 187-200.

McGeoch, M. A., Van rensburg, B. J., Botes, A. (2002). The verification and application of bioindicators: A case study of dung beetles in a savanna ecosystem. Journal of Applied Ecology. 39: 661-672.

Medina, C. A. \& Pulido, L. A. (2009). Escarabajos coprófagos (Coleoptera: Scarabaeinae) de la Orinoquía colombiana. Biota Colombiana. 10 (1 y 2): 55-62.

Medina, C. A. \& Lopera-Toro, A. (2000). Clave ilustrada para la identificación de los géneros de escarabajos coprófagos (Coleoptera: Scarabaeinae) de Colombia. Caldasia. 22 (2): 299-315.

Medina, C. A., Lopera, A., Vitolo, A., Gill, B. (2001). Escarabajos coprófagos (Coleoptera: Scarabaeidae: Scarabaeinae) de Colombia. Biota Colombiana. 2 (2):131-144.

Miles, L., Newton, A. C., De Fries, R. S., Ravilious, C., May, I., Blyth, S., Kapos, V., Gordon, J. E. (2006). A global overview of the conservation status of tropical dry forests. Journal of Biogeography. 33 (3): 491-505.

Montes-Correa, A., Jiménez, J. D., Vergara, D., Ávila, Y., Saboyá, L., Renjifo, J. M. (2015). Herpetofauna del campus de la Universidad del Magdalena, Santa Marta, Colombia. Revista de Biodiversidad Neotropical. 5 (1): 54-63.

Montes De Oca, E. \& Halffter, G. (1995). Daily and seasonal activities of a guild of the coprophagous, burrowing beetle (Coleoptera: Scarabaeidae: Scarabaeinae) in tropical grassland. Tropical Zoology. 8: 159-180.
Murphy, P. G. \& Lugo, A. E. (1986). Ecology of tropical dry forest. Annals Review of Ecology and Systematics. 17: 67-68.

Navarro, I. L., Roman, A. K., Gómez, F. H., Pérez, H. A. (2011). Listado de escarabajos coprófagos (Coleoptera: Scarabaeidae: Scarabaeinae) de la Serranía de Coraza, Sucre (Colombia). Revista Colombiana de Ciencia Animal. 3 (2): 262-268.

Nichols E., Larsen, T., Spector, S., Davis, A. L., Escobar, F., Favila, M., Vulinec, K., The Scarabaeinae Research Network. (2007). Global dung beetle response to tropical forest modification and fragmentation: A quantitative literature review and meta-analysis. Biological Conservation. 137 (1): 1-19.

Noriega, J. A. (2015). How a locality can have so many species? A case study with dung beetles (Coleoptera: Scarabaeinae) in a tropical rain forest in Colombia. 175-204 pp. In: Beetles: Biodiversity, ecology and role in the environment. C. Stack (Ed.), Nova Science Publishers, Inc. 264 p.

Noriega, J. A. \& Fagua, G. (2009). Monitoreo de escarabajos coprófagos (Coleoptera: Scarabaeidae) en la región neotropical. En: A. Acosta, G. Fagua, A.M. Zapata (Eds.). Técnicas de campo en ambientes tropicales: Manual para el monitoreo en ecosistemas acuáticos y artrópodos terrestres. Unidad de Ecología y Sistemática - UNESIS, Pontificia Universidad Javeriana, Bogotá. 215 p.

Noriega, J. A., Solís, C., Escobar, F., Realpe, E. (2007). Escarabajos coprófagos (Coleoptera: Scarabaeidae) de la provincia de la Sierra Nevada de Santa Marta. Biota Colombiana 8 (1): 77-86.

Noriega, J. A., Renjifo, J. M., Vaz-de-Mello, F. (2008). First report of the genus Tetramereia Klages, 1907 (Coleoptera: Scarabaeidae: Phanaeini) in Colombia - notes to its distribution. Biota Colombiana. 9 (1): 131-133.

Noriega, J. A., Moreno, J., Otavo, S. (2011). Quince años del arribo del escarabajo coprófago Digitonthophagus gazella (Fabricius, 1787) (Coleoptera: Scarabaeidae) a Colombia: proceso de invasión y posibles efectos de su establecimiento. Biota Colombiana. 12 (2): 35-44.

Noriega, J. A., Solís, C., García, H., Murillo-Ramos, L., Renjifo, J. M., Olarte, J. E. (2013). Sinopsis de los escarabajos coprófagos (Coleoptera: Scarabaeinae) del Caribe colombiano. Caldasia. 35 (2): 465-477.

Otavo, S., Parrado-Rosselli, A., Noriega, J. A. (2013). Superfamilia Scarabaeoidea (Insecta: Coleoptera) como elemento bioindicador de perturbación antropogénica en un parque nacional amazónico. Revista de Biología Tropical. 61 (2): 735-752.

Pizano, C. \& García, H. (2014). El bosque seco tropical en Colombia. Instituto de Investigación de Recursos Biológicos Alexander von Humboldt (IAvH). Bogotá, D.C., Colombia. 353 p.

Prado, D. E. \& Gibbs, P. E. (1993). Patterns of species distributions in the dry seasonal forests of South America. Annals of the Missouri Botanical Garden. 80: 902-927. 
Prance, G. T. (2006). Tropical savannas and seasonally dry forests: An introduction. Journal of Biogeography. 33: 385-386.

Pulido, L. A., Medina, C. A., Riveros, R. A. (2007). Nuevos registros de escarabajos coprófagos (Scarabaeidae: Scrabaeinae) para la región Andina de Colombia. Parte I. Revista de la Academia Colombiana de Ciencias Exactas, Físicas y Naturales. 31 (119): 305-310.

Quintero, I. \& Halffter, G. (2009). Temporal changes in a community of dung beetles (Insecta: Coleoptera: Scarabaeinae) resulting from the modification and fragmentation of tropical rain forest. Acta Zoológica Mexicana (n. s.). 25 (3): 625-649.

Rangel, O. \& Garzón, A. (1995). Sierra Nevada de Santa Marta (Colombia). Cap. 10. 155-170 pp. En: Rangel, O. (Ed.). Colombia Diversidad Biótica I. Instituto de Ciencias Naturales. 442 p.

Rivera, C. \& Wolff, M. (2007). Digitonthophagus gazella (Coleoptera: Scarabaeidae): distribución en América y dos nuevos registros para Colombia. Revista Colombiana de Entomología. 33 (2): 190-192.

Rojano, K. \& España, C. (2013). Composición y estructura vegetal presente en la parcela permanente del campus de la Universidad del Magdalena, Santa Marta, Colombia. Trabajo de grado. Programa de Biología, Facultad de Ciencias Básicas, Universidad del Magdalena.

Shahabuddin, C., Shulze, H., Tscharntke, T. (2005). Changes of dung beetle communities from rainforests towards agroforestry systems and annual cultures. Biodiversity and Conservation. 14: 863-877.
Solís, A. \& Kohlmann, B. (2002). El género Canthon (Coleoptera: Scarabaeidae) en Costa Rica. Giornale Italiano di Entomologia. 10: 1-68.

Solís, A. \& Kohlmann, B. (2004). El género Canthidium (Coleoptera: Scarabaeidae) en Costa Rica. Giornale Italiano di Entomologia. 52 (11): 1-73.

Solís, A. \& Kohlmann, B. (2013). El género Uroxys (Coleoptera: Scarabaeidae) en Costa Rica. Giornale Italiano di Entomologia. 13 (58): 289-340.

Solís C., Noriega, J. A., Herrera, G. (2011). Escarabajos coprófagos (Coleoptera: Scarabaeinae) en tres bosques secos del departamento del Atlántico-Colombia. Boletín del Museo de Entomología de la Universidad del Valle. 12 (1): 33-41.

Strewe R., Villa, C., Alzate, J., Beltrán, J., Moya, J., Navarro, C., Utría, G. (2009). Las aves del campus de la Universidad del Magdalena, Santa Marta, Colombia. Revista Intropica. 4: 79-91.

Torrijos, P. \& Cuadrado, B. (2008). Inventario florístico de la granja de la Universidad del Magdalena. Facultad de Ciencias Básicas, Universidad del Magdalena. 18 p.

UNESCO. (1973). International classification and mapping of vegetation. Ecology and conservation publication, No. 6 . Unesco, Paris. 93 p.

Vaz-De-Mello, F. Z., Edmonds, W. D., Ocampo, F. C., Schoolmeesters P. (2011). A multilingual key to the genera and subgenera of the subfamily Scarabaeinae of the New World (Coleoptera: Scarabaeidae). Zootaxa. 2854: 1-73. 